\title{
Korean Clinical Practice Guideline for the Diagnosis and Treatment of Insomnia in Adults
}

\author{
Hayun Choi', Soyoung Youn², Yoo Hyun Um³ ${ }^{3}$, Tae Won Kim4, Gawon Ju', Hyuk Joo Lee ${ }^{6}$, Chungsuk Lee ${ }^{2}$, \\ Sang Don Lee ${ }^{7}$, Kyungyeol Bae ${ }^{8}$, Seong Jae Kim ${ }^{9}$, Ji Hyun Lee ${ }^{10}$, Tae Kim ${ }^{11}$, and Seockhoon Chung ${ }^{12} \otimes$ \\ ${ }^{1}$ Department of Psychiatry, Veteran Health Service Medical Center, Seoul, Republic of Korea \\ ${ }^{2}$ Clearspring Psychiatric Clinic, Seoul, Republic of Korea \\ ${ }^{3}$ Department of Psychiatry, College of Medicine, The Catholic University of Korea, Seoul, Republic of Korea \\ ${ }^{4}$ Department of Psychiatry, St. Vincent's Hospital, The Catholic University of Korea, Suwon, Republic of Korea \\ ${ }^{5}$ Department of Psychiatry, Chungbuk National University Hospital, Cheongju, Republic of Korea \\ ${ }^{6}$ Department of Psychiatry, Ulsan University Hospital, Ulsan, Republic of Korea \\ ${ }^{7}$ Department of Psychiatry, Armed Forces Capital Hospital, Seongnam, Republic of Korea \\ ${ }^{8}$ Department of Psychiatry, Damyang Chamsarang Hospital, Damyang, Republic of Korea \\ ${ }^{9}$ Department of Psychiatry, Doeun Hospital, Jincheon, Republic of Korea \\ ${ }^{10}$ Department of Psychiatry, Dream Sleep Clinic, Seoul, Republic of Korea \\ ${ }^{11}$ Department of Biomedical Science and Engineering, Gwangju Institute of Science and Technology, Gwangju, Republic of Korea \\ ${ }^{12}$ Department of Psychiatry, Asan Medical Center, University of Ulsan College of Medicine, Seoul, Republic of Korea
}

Objective We aim to present a clinical guideline for the diagnosis and treatment of insomnia in adults by reviewing and integrating existing clinical guidelines. The purpose of this guideline is to assist clinicians who perform evidence-based insomnia treatment.

Methods We selected literature that may be appropriate for use in guideline development from evidence-based practice guidelines that have been issued by an academic or governmental institution within the last five years. The core question of this guideline was made in sentence form including Patient/Problem, Intervention, Comparison, Outcome (PICO) elements. After searching PubMed, EMBASE, and medical guideline issuing agencies, three guidelines were judged to be the most appropriately reviewed, up-to-date, and from trusted sources.

Results The Appraisal of Guidelines for Research and Evaluation (AGREE) II tool was used to evaluate the quality of the three clinical guidelines. The final outcome of the guideline development process is a total of 15 recommendations that report the strength of the recommendation, the quality of evidence, a summary of content, and considerations in applying the recommendation.

Conclusion It is vital for clinical guidelines for insomnia to be developed and continually updated in order to provide more accurate evidence-based treatments to patients.

Psychiatry Investig 2020;17(11):1048-1059

Key Words Insomnia, Guideline, CBT-I, Sleep.

\section{INTRODUCTION}

Insomnia is an independent disease, while it is also a symptom caused by or closely related to other physical or mental illnesses and sleep disorders. Insomnia often occurs tempo-

Received: April 22, 2020 Revised: August 6, 2020

Accepted: October 7, 2020

$\triangle$ Correspondence: Seockhoon Chung, MD, PhD

Department of Psychiatry, University of Ulsan College of Medicine, Asan Medical Center, 88 Olympic-ro 43-gil, Songpa-gu, Seoul 05505, Republic of Korea

Tel: +82-2-3010-3411, Fax: +82-2-485-8381, E-mail: schung@amc.seoul.kr

(a) This is an Open Access article distributed under the terms of the Creative Commons Attribution Non-Commercial License (https://creativecommons.org/licenses/bync/4.0) which permits unrestricted non-commercial use, distribution, and reproduction in any medium, provided the original work is properly cited. rarily and then improves; it may become chronic due to poor sleep habits, conditional hyperactivity, and cognitive distortions about sleep.

In this guideline, the diagnostic criteria of chronic insomnia were based on definition of "chronic insomnia disorder" of the International Classification of Sleep Disorders (ICSD) ${ }^{1}$ and "insomnia disorder" of the Diagnostic and Statistical Manual of Mental Disorders-fifth edition (DSM-5). ${ }^{2}$ These diagnostic criteria are similar in that they define insomnia based on difficulty starting and maintaining sleep, or early morning awakening, causing significant distress or impairment more than three times a week, for at least 3 months.

The American Academy of Sleep Medicine (AASM) ${ }^{1}$ and the 
European Sleep Research Society (ESRS) ${ }^{2}$ recommend first-line treatment for insomnia with cognitive behavioral therapy for insomnia (CBT-I) based on the meta-analysis results. If individuals have difficulty participating in CBT-I, or if CBT-I leads to no improvement in symptoms, hypnotic medications may be considered ${ }^{3,4}$ Hypnotic medications can help to resolve a patient's insomnia, but can cause side effects such as abuse and/ or dependence, falls, and delirium. In addition, many patients who take sleeping pills report that they still are unable to sleep properly. A study by the Korean Health Insurance Review and Assessment Service in 2011 Among people who have taken triazolam or zolpidem once, $5 \%$ of people take more than one tablet every day per year. And some patients have been prescribed unusually high amounts of sleeping pills. ${ }^{5}$

It is vital for sleep medication regimens to be personalized and optimized to patient's, but there are currently no clinical guidelines related to insomnia registered with the Korean Medical Guideline Information Center (KoMGI) ${ }^{6}$ as of February 2018. On the contrary, many clinical guidelines for the treatment of insomnia have already been developed and distributed abroad (Supplementary Table 1 in the online-only Data Supplement). Therefore, it is necessary to develop and disseminate a guideline for insomnia in order to help clinicians implement more appropriate insomnia treatments.

\section{METHODS}

\section{Working committee \& advisory committee}

In March 2018, a working committee was formed to develop the Korean clinical guidelines for the diagnosis and treatment of insomnia, sponsored by the Korean Neuropsychiatric Association. The working committee, composed of specialists who have extensive experience in academic activities and clinical practice in the field of sleep medicine The working committee consists of 15 neuropsychiatrists, four of whom worked at university hospitals, three worked at private clinics, two worked at general hospitals, two worked at psychiatric hospitals, and one worked at a university. The Working Committee prepared an adaptation plan, decides on key data sources, created key questions, investigated for international guidelines, evaluated key medical guidelines for adaptation, established a comparison table of recommendations, prepared a draft acceptance development, undertook a report on the process and the final guideline based on external review and consultation.

An advisory committee comprised of multidisciplinary specialists: 13 neuropsychiatrists, 2 neurologists, 2 preventive medicine specialists, 1 family medicine specialist, 1 otolaryngologist, and 1 psychologist. Nine of the neuropsychiatrists worked at a university hospital, three worked at a private hospital, and one worked at a psychiatric hospital. Other departmental spe- cialists worked in university hospitals, and the psychologist worked in a university. The advisory committee selected the subject of the guideline, checked the feasibility of adaptation, decided on the consensus process, prepared criteria for inclusion and exclusion of treatment guidelines, decided on subject areas, and reviewed the draft guideline.

\section{Developmental process}

The goals of this process were as follows: first, to develop guidelines for insomnia treatment through systematic review and synthesis of existing insomnia treatment guidelines; second, to review the evidence for non-pharmaceutical treatments and develop integrated treatment guidelines; and third, to update the existing guidelines with information about new drugs identified through systematic review.

This guideline primarily addresses the differential diagnosis and treatment of insomnia disorders for men and women aged 19 or older. The treatments for insomnia disorders are provided in the form of evidence-based medical guidance. The purpose of the guideline is to help the clinician establish the best treatment strategy for each clinical case based on existing evidence. The development phase of this guidance is presented in Figure 1 in three phases: set-up, adaptation, and finalization according to the ADAPTE process (Figure 1). ${ }^{7}$

\section{Adaptation}

The scope of this guideline was described using the Population, Intervention, Professionals, Outcomes, Healthcare settings (PIPOH) tool ${ }^{7}$ is as follows (Supplementary Table 2 in the online-only Data Supplement). The key question in this guideline was based on Patient/Problem, Intervention, Comparison, Outcome (PICO) elements, ${ }^{8}$ and the final draft of the

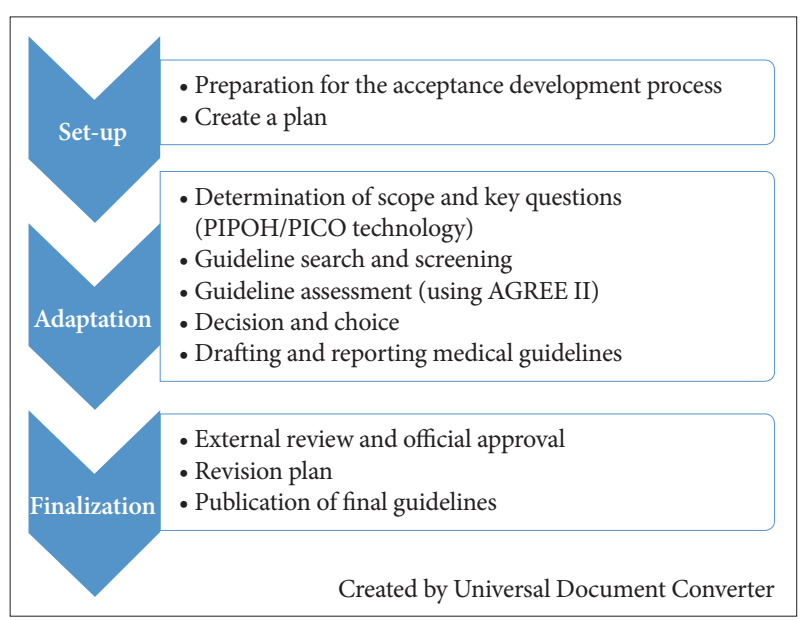

Figure 1. Development of the Korean clinical practice guideline for the diagnosis and treatment of insomnia. PIPOH: Population, Intervention, Professionals, Outcomes, Healthcare setting, PICO: Patient/Problem, Intervention, Comparison, Outcome, AGREE: Appraisal of Guidelines for Research and Evaluation. 
search terms including PICO was drafted by the second working committee and advisory committee (June 15, 2018).

\section{Selection and evaluation of existing clinical guidelines}

The criteria for selecting documents that could be used to develop the present guideline were set by the first Medical Guidelines Development working committee. The selection criteria included evidence-based medical guidelines issued by a research society or a government agency within the past five years. PubMed, EMBASE, and various medical guideline such as the National Guideline Clearinghouse (NGC), National institute for health and care excellence (NICE), and Guidelines International Network (GIN).

The search results were reviewed at a meeting of the working committee and advisory committee together (June 15, 2018), Guidelines were evaluated to ensure that they were appropriate for Korean sleep medicine experts, up-to-date, and issued by reputable institutions. The AASM, ESRS, and the American College of Physicians (ACP) guidelines were considered to be most relevant. ${ }^{3,49}$ The three selected guidelines were evaluated by four independent raters on several aspects using the Appraisal of Guidelines for Research and Evaluation (AGREE) II tool, ${ }^{10}$ including quality, rigor of development, content, and applicability. The AGREE $\mathrm{II}^{10}$ tool is composed of 23 detailed evaluation items in 6 evaluation areas, and each item is rated on a 7-point Likert scale. It was found that each of the three guidelines had their own characteristics that complemented each other. Recommendations for CBT-I, a non-pharmacological treatment for insomnia, were given by the ACP Treatment Guideline (2016); ${ }^{4}$ for the overall diagnosis and treatment of insomnia by the ESRS Treatment Guidelines (2017); ${ }^{2}$ and for each drug based on a detailed level of evidences by the AASM Treatment Guideline (2017). ${ }^{1}$ Therefore, instead of selecting a single guideline, the Working Committee decided to develop the Korean clinical guideline based on the three guidelines that reflects the various opinions of different countries.

\section{Determination and selection of recommendations}

The ACP's evidence-level evaluation system $(2016)^{9}$ (Supplementary Table 3 in the online-only Data Supplement) used the Grading of Recommendations Assessment, Development and Evaluation (GRADE) $)^{11,12}$ system. We evaluated whether the rationale of the ACP guideline contained in each study was reliable and correct and whether appropriate investigations were made with regard to possible side effects.

The ESRS guidelines (2017) ${ }^{3}$ also used the GRADE ${ }^{11,12}$ system (Supplementary Table 4 in the online-only Data Supplement). When evaluating the level of evidence, the ESRS treatment guidelines determine the level of evidence as high quality when it was determined that the results would not change signifi- cantly in the existing meta-study, even if a follow-up study was conducted. In contrast, the level of evidence is determined to be low quality when the results of the meta-study were uncertain.

The AASM Guideline (2017) ${ }^{3}$ confirmed the application of the GRADE ${ }^{11,12}$ system on the evidence level and discusses how this system was used (Supplementary Table 5 in the online-only Data Supplement). The AASM Guideline also sets the initial level of evidence, with and without a randomized clinical trial (RCT) and then reduces it to account for the risk of bias, inconsistency, indirectness, inaccuracy, and publication bias. If the level does not need to be reduced, or if there is a large consistent effect, a good dose-response gradient, or if all confounding factors are well considered, then the level is elevated. In addition, there are two types of recommendation, strong and weak; strong recommendations mean that clinicians follow most situations when the drug is indicated, while recommendations for low levels indicate uncertain adequacy.

We determined that the level of recommendation and level of evidence in this guideline was to follow those established in the above three guidelines. Since each medical guideline had already been assessed for quality of evidence using the GRADE $^{11,12}$ system, this guideline accepted the evidence level of each medical guideline. The recommendation grades were classified into two categories, strong and weak, and if the three guidelines differed, a consensus was reached through discussion of the working committee (Supplementary Table 6 in the online-only Data Supplement).

\section{Drawing, drafting, and finalizing of recommendations}

The working committee drafted recommendations by extracting recommendations on key questions from the AASM, ESRS, and ACP guidelines. Subsequently, the working committee conducted a preliminary discussion on the acceptability, applicability, and clarity of the recommendations, and proposed amendments through informal agreement between the working committees. Based on the recommendations adopted, the working committee divided the topics into drafting guidelines. Following the drafting of recommendations, the advisory committee reviewed and approved the draft. The final version of this guideline has been certified by the Review Committee of the Korean Neuropsychiatric Association.

\section{RESULTS}

\section{Diagnosis}

\section{Clinical history taking and lab tests}

Diagnosis of insomnia is mainly based on symptoms reported by the patient. Therefore, detailed history taking is vital. The recommendations for insomniac diagnostic assessment items 
according to the ESRS Guideline are presented with the author's consent (Table 1). ${ }^{4}$

\section{Assessment tools for sleep disorders, including insomnia}

\section{Polysomnography}

Polysomnography is not necessarily recommended for acute or chronic insomnia. ${ }^{13}$ Although polysomnography has recently been used as a supplemental tool, it has been limited to obstructive sleep apnea narcolepsy and subjective insomnia without objective evidence of insomnia. ${ }^{13}$

\section{Actigraphy}

The actigraph is a compact motion detector that can be worn on the wrist for days to months. The activity recorder does not measure sleep itself, but indirectly assesses sleep status by measuring activity. In the presence of other sleep indicators, such as a sleep diary, sleep status can be assessed more objectively and reliably through actigraphy. ${ }^{14}$

\section{Sleep diaries}

A sleep diary is a way of evaluating insomnia by manually recording information in the morning about sleep the night before. Factors that are important for the evaluation and treatment of insomnia, such as bedtime, wake-up time, sleep latency, and nighttime awakening frequency should be recorded. In addition, the individual's sleep patterns can be calculated quantitatively, which is useful for evaluating change over the course of insomnia treatment.

\section{Questionnaires evaluating sleep disorders}

Table 2 briefly introduces the most commonly used clinically useful tools related to sleep disorders, including insomnia. The Insomnia Severity Index (ISI) ${ }^{15}$ is 7 -item self-rating scale to assess the patient's perception of insomnia. The Pittsburgh Sleep Quality Index (PSQI ${ }^{16}$ is 19-item self-report measure of general sleep quality (poor sleep: global score $>5$ ). PSQI evaluates seven areas, including duration of sleep, sleep disturbance, sleep-onset latency, daytime dysfunction caused by

Table 1. Diagnostic management of insomnia and its co-morbidities

1. Medical history and examination (strong recommendation)

- The anamnesis should include caregivers if necessary

- Former and present somatic disorders (including pain)

- Substance use (medication, alcohol, caffeine, nicotine, illegal drugs)

- Physical examination

- Additional measures (if indicated): laboratory testing including, e.g. blood count, thyroid, hepatic and renal parameters, CRP, hemoglobin, ferritin and vitamin B12, ECG, EEG, CT/MRT, circadian markers (melatonin, core, body temperature)

2. Psychiatric/psychological history (strong recommendation)

- Former and present mental disorders

- Personality factors

- Work and partnership situation

- Interpersonal conflicts

3. Sleep history (strong recommendation)

- History of the sleep disorder, including triggering factors

- Information from bed partner (periodic limb movements during sleep, pauses in breathing)

- Work time/circadian factors (shift- and night-work, phase advance, delay)

- Sleep-wake pattern, including daytime sleep (sleep diary, sleep questionnaires)

4. Actigraphy

- In case of clinical suspicion of irregular sleep-wake schedules or circadian rhythm disorders (strong recommendation)

- To assess quantitative sleep parameters (weak recommendation)

5. Polysomnography

- In case of clinical suspicion of other sleep disorders like periodic limb movement disorder, sleep apnea or narcolepsy (strong recommendation)

- Treatment-resistant insomnia (strong recommendation)

- Insomnia in occupational at-risk groups, e.g. professional drivers (strong recommendation)

- In case of clinical suspicion of large discrepancy between subjectively experienced and polysomnographically measured sleep (strong recommendation)

Ref $^{4}$ with permission from the author. CRP: C-reactive protein, CT: computed tomography, ECG: electrocardiogram, EEG: electroencephalogram, MRT: magnetic resonance tomography 
Table 2. List of survey tools that are used primarily in the evaluation of sleep disorders

\begin{tabular}{|c|c|}
\hline \multicolumn{2}{|l|}{ Insomnia } \\
\hline Insomnia Severity Index ${ }^{15}$ & 7-item rating scales to assess the patient's perception of insomnia \\
\hline Pittsburgh Sleep Quality Index ${ }^{16}$ & 19-item rating scale of sleep quality (poor sleep: global score >5) \\
\hline $\begin{array}{l}\text { Dysfunctional Beliefs and Attitudes about Sleep } \\
\text { scales }^{17}\end{array}$ & $\begin{array}{l}\text { 30-item original or 16-item short scale for assessing dysfunctional thoughts about } \\
\text { sleep }\end{array}$ \\
\hline \multicolumn{2}{|l|}{ Daytime sleepiness } \\
\hline Epworth Sleepiness Scale ${ }^{18}$ & 8 -item questionnaire to assess subjective sleepiness $(0-24$; normal $<10)$ \\
\hline Stanford Sleepiness Scale ${ }^{19}$ & 7-point Likert-type scale has descriptors to assess daytime sleepiness \\
\hline \multicolumn{2}{|l|}{ Sleep-disordered breathing } \\
\hline STOP, ${ }^{20}$ STOP-Bang ${ }^{21}$ & 8-item rating scale to screen patients with obstructive sleep apnea \\
\hline Berlin Questionnaire $^{22}$ & 10-item questionnaire to identify patients with obstructive sleep apnea \\
\hline \multicolumn{2}{|l|}{ Chronotype } \\
\hline Morningness-Eveningness Questionnaire ${ }^{23}$ & 19-item rating scale to measure a person's circadian rhythm (early birds or night owls) \\
\hline \multicolumn{2}{|l|}{ General assessment } \\
\hline SLEEP-50 $0^{24}$ & 50-item rating scale to detect various sleep disorders \\
\hline
\end{tabular}

STOP: snoring, tiredness during daytime, observed apnea, and high blood pressure

sleepiness, sleep efficiency, sleep medication needs, and overall sleep quality. Dysfunctional Beliefs and Attitudes about Sleep scales (DBAS) ${ }^{17}$ is 30 -item original or 16 -item short scale for assessing negative cognitions about sleep. Overcoming these existing cognitions is the goal of cognitive behavior therapy for insomnia.

Epworth Sleepiness Scale (ESS) ${ }^{18}$ is used to assess self-rated average sleepiness over usual way of life in recent times (0-24; normal $<10)$. Stanford Sleepiness Scale $(\mathrm{SSS})^{19}$ is a 7-point Likert-type scale and has descriptors to assess daytime subjective sleepiness. STOP, ${ }^{20}$ STOP-Bang ${ }^{21}$ are rating scales to screen patients with obstructive sleep apnea. The STOP questionnaire consists of 4 questions about snoring, tired, observed, blood pressure. The STOP-BANG questionnaire additionally includes 4 questions about body mass index, age, neck circumference, and gender. Berlin Questionnaire (BQ) 22 is a 10-item questionnaire to identify patients with obstructive sleep apnea. It includes the following: snoring and witnessed apnea, being sleepy more than three or four times a week, and the presence of hypertension. Morningness-Eveningness Questionnaire (MEQ $)^{23}$ is composed of 9-item rating scales to measure a person's circadian rhythm. SLEEP-50 ${ }^{24}$ is a 50 -item rating scale to detect various sleep disorders

\section{Process for diagnosis of insomnia}

First, a medical history and physical examination related to insomnia, psychiatric evaluation, and sleep evaluation should be performed. As shown in Figure 2, various sleep disorders that exhibit symptoms similar to insomnia, such as circadian rhythm sleep-wake disorder, obstructive sleep apnea, restless legs syndrome, and parasomnia, should be differentially diag- nosed. It is necessary to rule out other sleep disorders through the use of a sleep diary, actigraphy, and polysomnography (Figure 2).

\section{Treatment}

\section{Non-pharmacologic treatments}

\section{Cognitive behavioral therapy for insomnia (CBT-I)}

The efficacy of non-pharmacologic treatments for insomnia were reaffirmed in the 2008 American Society for the Evaluation and Treatment of Adult Insomnia report, ${ }^{25}$ and CBT-I has been recommended as the primary treatment for chronic insomnia in all sleep disorder treatment guidelines published in the US, Europe, and Australia since 2016. CBT-I corrects cognitive errors that lead to chronic insomnia and uses behavioral therapy to correct dysfunctional sleep-related habits that cause insomnia to persist. It has similar or better and longer lasting therapeutic effect after completion of treatment compared to that observed with drug therapy. ${ }^{25}$

In general, CBT-I includes: 1) education about normal sleep, sleep hygiene, and the purpose of CBT-I, 2) stimulus control therapy, 3) sleep restriction therapy, 4) relaxation techniques, and 5) cognitive therapy. ${ }^{26}$ It is common to deliver these elements across four to six sessions and allocate forty to sixty minutes for each session. ${ }^{27}$

\section{Pharmacologic treatments}

If a patient has difficulty in participating in CBT-I, or if CBT-I does not show any improvement in symptoms, clinicians may prescribe hypnotic medication only or with CBT-I. Meta-anal- 


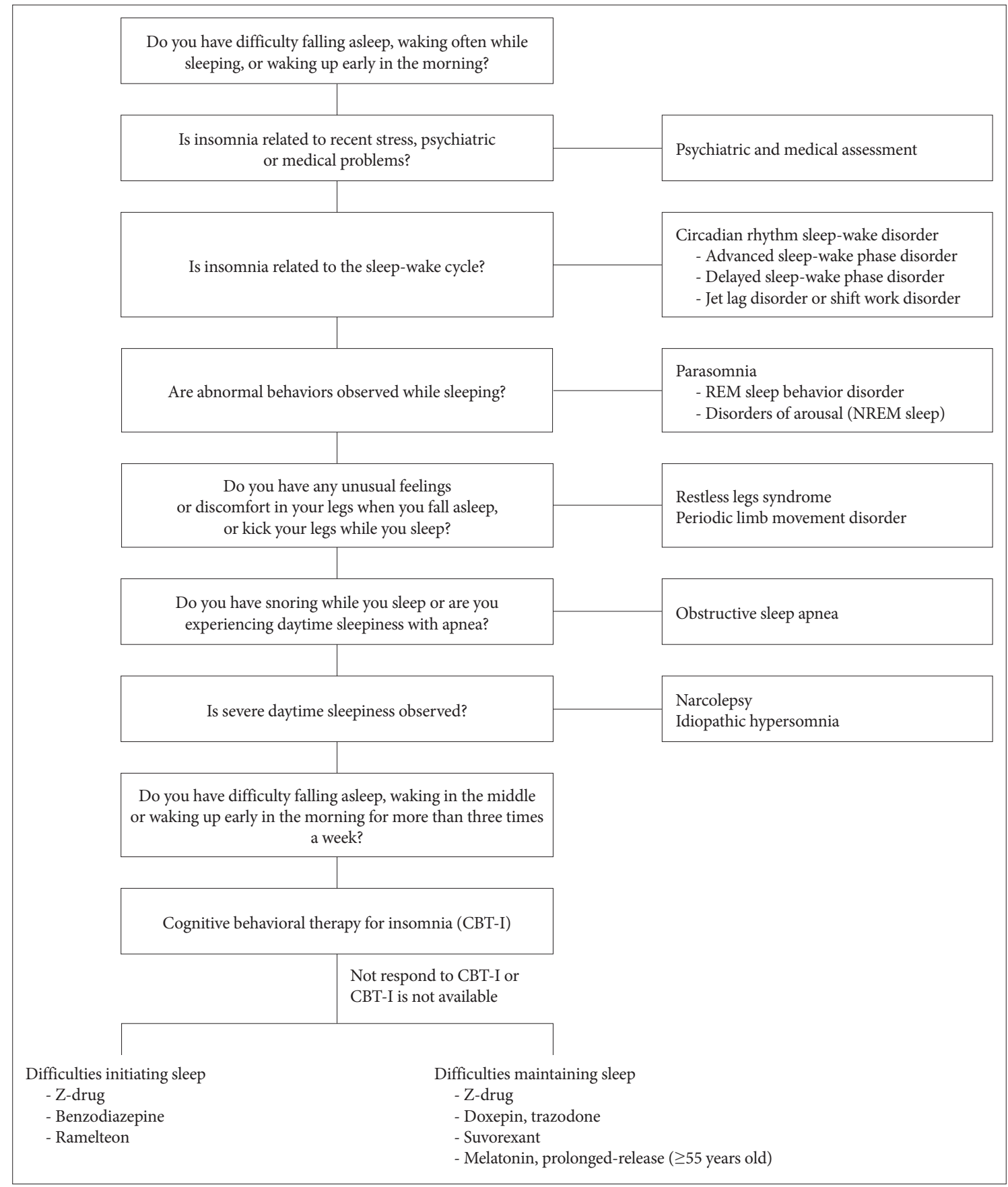

Figure 2. Flow chart of insomnia diagnosis. CBT-I: cognitive behavioral therapy for insomnia.

ysis for CBT-I provides a basis for effectiveness and safety and for the persistence of effects after treatment. ${ }^{1,2}$ In contrast, drug treatment is commonly used in Korea, but there is insufficient evidence for its effects and safety with long-term use. Therefore, clinicians should prescribe medication with caution, depending on the patient's characteristics and history of drug reactions, for as short a period as possible. Table 3 lists the drugs that are mainly used as sleeping pills in Korea. And the lists of recommendations in this guideline were in Table 4.

\section{Benzodiazepines}

Benzodiazepines act as a benzodiazepine (BDZ) receptor agonist in the gamma aminobutryric acid (GABA-A) receptor complex to promote the binding between GABA and GABA-A receptors, the most common inhibitory neurotransmitters of the central nervous system. ${ }^{28}$ Type $1 \mathrm{BDZ}$ receptors are related 
Table 3. Drugs used for insomnia in South Korea

\begin{tabular}{|c|c|}
\hline Drugs & Most common dosage \\
\hline \multicolumn{2}{|l|}{ A. Benzodiazepines } \\
\hline Flurazepam* & $15-30 \mathrm{mg}$ \\
\hline Triazolam* & $0.125-0.25 \mathrm{mg}$ \\
\hline Flunitrazepam* & $1 \mathrm{mg}$ \\
\hline Brotizolam* & $0.25 \mathrm{mg}$ \\
\hline Clonazepam & $0.5 \mathrm{mg}$ \\
\hline \multicolumn{2}{|l|}{ B. Non-benzodiazepine } \\
\hline \multicolumn{2}{|c|}{ Non-benzodiazepine GABA modulator (z-class) } \\
\hline Zolpidem immediate-release* & $5-10 \mathrm{mg}$ \\
\hline Zolpidem controlled-release* & $6.25-12.5 \mathrm{mg}$ \\
\hline Eszopiclone* & $1-3 \mathrm{mg}$ \\
\hline \multicolumn{2}{|l|}{ Antidepressants } \\
\hline Trazodone & $25-50 \mathrm{mg}$ \\
\hline Mirtazapine & $7.5-30 \mathrm{mg}$ \\
\hline Amitriptyline & $10-30 \mathrm{mg}$ \\
\hline Doxepin* & $3-6 \mathrm{mg}$ \\
\hline \multicolumn{2}{|l|}{ Antihistamines } \\
\hline Doxylamine* & $25 \mathrm{mg}$ \\
\hline Diphenhydramine* & $25-50 \mathrm{mg}$ \\
\hline \multicolumn{2}{|l|}{ Melatonin } \\
\hline Prolonged-release melatonin* & $2 \mathrm{mg}$ \\
\hline
\end{tabular}

*approved by Korean Food and Drug Administration (KFDA). GABA: $\gamma$-aminobutyric acid

to sleep, and type 2 receptors are associated with muscle relaxation and psychomotor dysfunction and have anticonvulsive effects. ${ }^{28}$ Type $3 \mathrm{BDZ}$ receptors are associated with withdrawal symptoms or resistance. ${ }^{28}$ Benzodiazepines are the third most prescribed insomnia treatment drugs in the US, after benzodiazepine receptor agonists (BzRAs) such as Z-class drugs and trazodone, an antidepressant. ${ }^{29}$ Currently, the following benzodiazepines are approved by the Korean Food and Drug Administration (KFDA) for short-term insomnia treatment (up to 4 weeks): flurazepam, triazolam, flunitrazepam, and brotizolam..$^{30}$ Benzodiazepines, such as lorazepam, clonazepam, diazepam, and etizolam, have not been approved by the KFDA, but are used off-label for the treatment of insomnia in clinical settings. Clinicians typically consider prescribing shortacting benzodiazepins for sleep initiation insomnia, and longacting benzodiazepines for early morning awakening or sleep maintenance problems.

According to the AASM guideline (2017), ${ }^{3}$ studies have shown that benzodiazepins reduce sleep latency, increase total sleep time, decrease wakefulness during sleep, and improve sleep quality. The AASM guideline $(2017)^{3}$ concludes that the side effects of benzodiazepines outweigh the treatment effects, and that there is insufficient data to verify the effects of benzodiazepines for treatments lasting over four weeks.

The ESRS guideline $(2017)^{4}$ also strongly recommends that patients who take daily doses of benzodiazepines should be instructed to take them intermittently when necessary. Although benzodiazepines have proven effective in short-term care lasting less than four weeks, their effects have not been sufficiently proven for long-term use, and the possibility of side effects remains high. The ACP guideline $(2016)^{9}$ states that there is not sufficient evidence for the effect of benzodiazepines on chronic insomnia in the general or elderly population, so benzodiazepines are recommended for a limited period of time if CBT-I does not have a sufficient effect.

Prolonged use of benzodiazepines can lead to resistance, dependence, and withdrawal symptoms if discontinued, especially in drugs with a short half-life, such as triazolam. ${ }^{28}$ It is desirable to start with the smallest dose and adjust based on improvement in symptoms and whether there are side effects. In particular, the prolonged use of benzodiazepines in the elderly may degrade cognitive function, and there is a high probability of falls and fractures. ${ }^{31}$

\section{Z-class drugs}

Z-class drugs are a type of BzRA called non-benzodiazepine hypnotics. Z-class drugs approved for use in insomnia by the KFDA are zolpidem and eszopiclone. Similarly to benzodiazepines, z-class drugs act on GABA A receptors but is designed to be selectively coupled to type 1 receptors. ${ }^{28}$ Studies on the effects of zolpidem have shown that zolpidem reduces sleep latency, increases, total sleep time, helps maintain sleep, and improves the quality of sleep. ${ }^{32}$ Common side effects of zolpidem include headaches, dizziness, and drowsiness, but the side effects are less common relative to benzodiazepines. ${ }^{33}$ However, zolpidem increases the occurrence of serious side effects such as sleepwalking or sleep-related eating disorders, loss of memory, hallucinations, and increased risk of suicide. ${ }^{34}$ Like benzodiazepines, zolpidem also carries a risk of resistance, dependence, and withdrawal.

The appropriate prescription dose for zolpidem immediate-release (IR) is 5 to $10 \mathrm{mg}$, and for zolpidem controlled-release (CR), an appropriate dose is 6.25 to $12.5 \mathrm{mg}$. It is recommended to prescribe $5 \mathrm{mg}$ zolpidem IR and $6.25 \mathrm{mg}$ zolpidem $\mathrm{CR}$ for long-term use, especially in older adults due to concerns about side effects. ${ }^{35}$

The AASM guideline $(2017)^{3}$ proposed the use of zolpidem in adults for sleep initiation and sleep maintenance disorders. The ESRS guideline (2017) $)^{4}$ shows that BzRAs with zolpidem have a similar effect as benzodiazepines in short-term use (i.e., less than four weeks). But, like benzodiazepines, prolonged use lasting more than four weeks is not recommended due to 
Table 4. List of recommendations for the Korean version of clinical practice guideline for the treatment of insomnia

\begin{tabular}{|c|c|c|c|}
\hline & Recommendation & $\begin{array}{l}\text { Strength of } \\
\text { recommendation }\end{array}$ & $\begin{array}{l}\text { Quality of } \\
\text { evidence }\end{array}$ \\
\hline 1 & $\begin{array}{l}\text { We recommend priority implementation of Cognitive Behavioral Treatment for insomnia (CBT-I) } \\
\text { in adults }\end{array}$ & Strong & Moderate \\
\hline 2 & $\begin{array}{l}\text { We recommend that clinicians consider using Zolpidem immediate-release (IR) as a treatment for } \\
\text { sleep initiation disorders in adults }\end{array}$ & Weak & Low \\
\hline 3 & $\begin{array}{l}\text { We recommend that clinicians consider using Zolpidem controlled-release (CR) as a treatment for } \\
\text { sleep initiation and maintenance disorders in adults }\end{array}$ & Weak & Low \\
\hline 4 & $\begin{array}{l}\text { We recommend that clinicians consider using eszolpiclon as a treatment for sleep initiation } \\
\text { disorders in adults }\end{array}$ & Weak & Low \\
\hline 5 & $\begin{array}{l}\text { We recommend that clinicians consider using Zaleplon as a treatment for sleep initiation disorders } \\
\text { in adults }\end{array}$ & Weak & Low \\
\hline 6 & $\begin{array}{l}\text { We recommend that clinicians consider using Triazolam as a treatment for sleep initiation } \\
\text { disorders in adults }\end{array}$ & Weak & Moderate \\
\hline 7 & $\begin{array}{l}\text { We recommend that clinicians consider using Doxepin as a treatment for sleep maintenance } \\
\text { disorders in adults }\end{array}$ & Weak & Low \\
\hline 8 & $\begin{array}{l}\text { We recommend that clinicians consider using Trazodone as a treatment for sleep maintenance } \\
\text { disorders in adults }\end{array}$ & Weak & Low \\
\hline 9.1 & $\begin{array}{l}\text { We recommend that clinicians consider not using Melatonin immediate-release agents to treat } \\
\text { insomnia in adults }\end{array}$ & Weak & Low \\
\hline 9.2 & $\begin{array}{l}\text { We recommend that clinicians consider using Melatonin prolonged-release agents for sleep } \\
\text { maintenance disorders in } 55 \text { years or older }\end{array}$ & & \\
\hline 10 & $\begin{array}{l}\text { We recommend that clinicians consider using Ramelteon as a treatment for sleep initiation } \\
\text { disorders in adults }\end{array}$ & Weak & Low \\
\hline 11 & $\begin{array}{l}\text { We recommend that clinicians consider using Suvorexant as a treatment for sleep maintenance } \\
\text { disorders in adults }\end{array}$ & Weak & Low \\
\hline 12 & We recommend that clinicians consider not using Doxylamine to treat insomnia in adults & Strong & Low \\
\hline 13 & We recommend that clinicians consider not using Diphenhydramine to treat insomnia in adults & Strong & Low \\
\hline 14 & We recommend that clinicians consider not using Valerian to treat insomnia in adults & Weak & Low \\
\hline 15 & $\begin{array}{l}\text { We recommend that clinicians consider educating patients to take benzodiazepines or } \\
\text { benzodiazepine receptor agonists in time for sleep prescribed during cognitive behavioral therapy }\end{array}$ & Weak & Low \\
\hline
\end{tabular}

concerns about side effects, and it is preferable to take it only intermittently if necessary rather than daily. ${ }^{4}$ The ACP guideline $(2016)^{9}$ also suggests short-term use of zolpidem for four to five weeks or less because of the lack of evidence related to the benefits and risks of long-term use.

\section{Melatonin and melatonin receptor agonist}

Melatonin is a hormone secreted by the pineal gland and is involved in regulating the circadian rhythm. Prolonged-release melatonin is the only prescribed melatonin product in Korea and has been approved for use in insomnia patients 55 years or older since 2014. Long-acting melatonin preparations are recommended to be taken two hours before going to bed. Unlike conventional melatonin preparations, which had a short half-life of 35 to 50 minutes and clears quickly in the body, the sustained-release agent maintains its concentration over 8-10 hours, mimicking the pattern of melatonin secretion in the body. ${ }^{36}$ In the US, fast-acting melatonin preparations are widely used as a dietary supplement without the need for a prescription.

Ramelteon is a melatonin receptor agonist that is approved by the US FDA for the treatment of insomnia, and is recommended by the AASM guideline (2017) ${ }^{3}$ for sleep initiation insomnia. According to the AASM guideline (2017), ${ }^{3}$ unlike ramelteon, the use of melatonin preparations for sleep initiation or maintenance insomnia is not recommended due to insufficient evidence of effectiveness. The ESRS guideline $(2017)^{4}$ and ACP guideline $(2016)^{9}$ do not recommend the use of melatonin in insomnia for the same reason. However, the above guidelines did not clearly distinguish sustained-release melatonin from fast-acting preparations. Sustained-release melatonin drugs do not act on GABA receptors, so they have fewer side effects such as decreased cognitive function, falls, rebound insomnia, dependence, tolerance, and withdrawal symptoms 
when compared to benzodiazepines or BzRAs.

Ramelteon is a melatonin $\mathrm{MT}_{1}$ and $\mathrm{MT}_{2}$ receptor agonist, and meta-analyses have shown some effects on reducing sleep latency and improving sleep quality and efficiency. However, this effect did not reach clinical significance and did not significantly increase total sleep time. Since no significant side effects were observed except daytime drowsiness, the risk to benefit ratio was considered to be excellent. The AASM guideline $(2017)^{3}$ recommends that it be used for sleep initiation insomnia, and the ACP guideline (2016) ${ }^{9}$ suggests that it can be useful for older patients. It is not yet commercially available in Korea.

\section{Orexin antagonists}

Orexin/hypocretin is a neuropeptide secreted from the perifornical area and orexin-producing neurons of the lateral hypothalamus and plays an important role in the maintenance of arousal. ${ }^{21}$ Blockade of orexin $\mathrm{OX}_{1}$ and $\mathrm{OX}_{2}$ receptors has been suggested to improve insomnia, resulting in the development of dual orexin receptor antagonists (DORAs) ${ }^{28} \mathrm{Su}-$ vorexant is a DORA that was approved by the US FDA as a treatment for insomnia in 2014. Two RCTs showed no significant clinical improvement in sleep latency and total sleep time, but clinically significant improvement was observed in sleep efficiency and sleep quality. ${ }^{3}$ No significant side effects were observed compared to placebo. ${ }^{3}$ The AASM guideline $(2017)^{3}$ proposed that suvorexant could be used for sleep maintenance disorders, and the ACP guideline $(2016)^{9}$ suggested that it could be used for sleep disorders. It is not yet utilized in Korea.

\section{Antidepressants}

With the development of several new forms of antidepressants, tricyclic antidepressants are not usually used for their antidepressant effects anymore, and are mainly used for pain or sleep control purposes. Doxepin is the only drug approved by the KFDA as a treatment for insomnia. The mechanism of action is believed to be antagonism of the histamine $\mathrm{H}_{1}$ receptor. Clinical studies of doxepin using $3 \mathrm{mg}$ and $6 \mathrm{mg}$ have reported significant improvements in wake time after sleep onset (WASO), total sleep time, and sleep efficiency compared to placebo. ${ }^{3}$ However, a meta-analysis showed that improvement of sleep initiation was not observed, though it was effective for sleep maintenance disorders. ${ }^{37}$ Diarrhea, drowsiness, and headaches are reported, but antidepressants have advantages in terms of fewer side effects compared to benzodiazepines or Z-drugs, and may be better suited for prescribing to the elderly due to having a relatively lower risk of falls.

Trazodone is a $5-\mathrm{HT}_{2 \mathrm{~A}}$ receptor antagonist ${ }^{38}$ and a $50 \mathrm{mg}$ dose shows antagonism of histamine $\mathrm{H}_{1}$ and $\alpha$-adrenergic receptors, acting as a sleeping pill. ${ }^{39}$ In insomnia, trazodone main- ly acts by reducing WASO and increasing total sleep time, sleep efficiency, and slow wave sleep; however, it has no effect on reducing sleep latency. ${ }^{40}$ Accordingly, it may be more beneficial for sleep maintenance disorders than sleep initiation disorders. Side effects such as headaches and postural hypotension can often occur, but the potential for abuse is relatively less. The AASM guideline $(2017)^{3}$ proposed not to use trazodone as a primary treatment for insomnia because there was no objective study that demonstrated its efficacy. In a meta-analysis study of trazodone published shortly after the publication of the AASM guideline, ${ }^{41}$ trazodone was reported to be effective and safe as an insomnia treatment.

\section{Antihistamines}

Antihistamines induce sleep through histamine $\mathrm{H}_{1}$ receptor antagonism. ${ }^{42}$ The Korean FDA has authorized the use of doxylamine and diphenhydramine for the purpose of supplemental treatment for insomnia. In the case of doxylamine, 25 $\mathrm{mg}$ is recommended but clinicians need to be careful when administering doxylamine to patients with severe myasthenia gravis, acute narrow angle glaucoma, or drug addiction. Diphenhydramine is approved at a dose of $50 \mathrm{mg}$ for the purpose of improving temporary insomnia, but it may be necessary to reduce it to $25 \mathrm{mg}$ depending on the patient. In Korea, doxylamine and diphenhydramine can be purchased at a pharmacy without prescription. Clinicians should be cautious for patients with asthma attacks, difficulty breathing due to chronic bronchitis, glaucoma, or lower urinary obstructive diseases such as prostate hypertrophy. The AASM guideline (2017) ${ }^{3}$ states that diphenhydramine improves sleep latency and total sleep time, but a meta-analysis has not yet been conducted. The ESRS guideline $(2017)^{4}$ recommends that antihistamines should not be used to improve insomnia.

\section{Phytotherapy (valerian, hops)}

Phytotherapy refers to the medicinal use of herbs, including valerian root which is dried and made into tea. It has long been used for the purpose of improving insomnia because of patients' rejection of the use of chemicals. Although studies have been conducted to verify the sleep improvement effects of herbs such as valerian and hops, they have not established clinical significance in the improvement of insomnia. ${ }^{43}$ Both the AASM guideline (2017) ${ }^{3}$ and ESRS guideline $(2017)^{4}$ do not recommend the use of valerian for insomnia.

\section{Selection of drugs according to clinical manifestations of insomnia}

\section{Sleep initiation disorders}

The Z-class drug, zolpidem IR formulation, can be used as 
a first-line treatment, and zolpidem CR can also be considered for sleep initiation disorders. Clinicians can also choose a BZD, such as triazolam. Although not yet introduced in Korea, ramelteon is also recommended for sleep initiation disorders.

\section{Sleep maintenance disorders}

Sleep maintenance disorders can be treated with zolpidem CR or antidepressants such as doxepin and trazodone. Patients 55 years or older can also use melatonin sustained-release formulation. Although not yet introduced in Korea, suvorexant is also recommended for sleep maintenance disorders.

\section{Timing of medication use}

The three guidelines ${ }^{3,49}$ provide no guidance on when to use sleeping pills. However, it is very important to consider the time that a patient takes sleeping pills. Patients usually want to fall asleep quickly right after taking sleeping pills. To optimize the effects of sleeping pills, patients need to take them at the "sleep time considering the sleep-wake cycle" not the "time patients want to sleep." In a study examining the relationship between timing of sleeping pills and subjective satisfaction, the satisfied group had a duration from administration of pills to wake up time (PTW) of 7.2 hours, and 9.3 hours in the unsatisfied group. ${ }^{44}$ Based on these findings, the same researchers suggested that sleeping pills be taken 7 hours before waking time instead of 30 minutes before bedtime in order to increase patient satisfaction. ${ }^{45}$ Since there are individual pharmacodynamic and pharmacokinetic differences, and limited evidence on their effects, these suggestions may not apply to all patients. It is important to stress that patients are likely to be dissatisfied with the instructions to take their medication 30 minutes before bedtime. Sustained-release melatonin is recommended to be taken one to two hours before going to bed. The concentration of the melatonin sustained-release agent peaks 1.6 to 2.6 hours after taking it. ${ }^{46}$ In consideration of the duration of action of the drug and sleep-wake cycles, clinicians can give instructions to take sleeping pills nine hours before morning wake-up time and to go to bed two hours later.

\section{DISCUSSION}

This guideline primarily addresses the differential diagnosis and treatment of insomnia disorders for men and women aged 19 years or older. This guideline focuses on the selection of drugs according to clinical patterns of insomnia and the timing of taking hypnotic medications. The original guidelines published in Korean in 2019 include the detailed processes of acceptance development, differential diagnosis of insomnia, tools for diagnosing sleep disorders, educational materials for the general public, and the format of a sleep diary. As the med- ical guidelines gradually contained different perspectives of each region and field of expertise, the three medical guidelines were finally combined and developed. The three selected guidelines were evaluated by four evaluators using the AGREE II tool in several aspects, including quality, being up-to-date, content, and acceptability/applicability.

As a final result of the acceptance development process, 15 recommendations were presented in consideration of the factors of Patient/Problem, Intervention, Comparison and Outcome (PICO) to enhance readability; these recommendations include recommended grades and levels of evidence, summary of content, and considerations when applying guidelines. Clinicians can refer to this guideline and easily apply it to patients. Neuropsychiatrists primarily led to the writing of these guidelines, but preventive medicine experts have also been consulted throughout. Furthermore, psychologists and various experts on sleep disorders, such as those working in the fields of neurology, family medicine, and otolaryngology, served as advisory committee members. The final version of the guideline was certified by the clinical guidance committee under the Korean Neuropsychiatric Association.

This guideline has several limitations. First, it may be somewhat insufficient to reflect the reality of Korea as it has accepted and developed three overseas medical guidelines. To overcome this limitation, the drugs currently used in Korea or those that are likely to be introduced in Korea have been discussed in this guideline, and KFDA-licensed medicines are listed. Second, although it is very important to provide information about the timing of medication, there remains a lack of data on the most effective timing. Third, there is some information on the termination of $\mathrm{BDZ}$ and $\mathrm{z}$-drug, but there is no mention of the termination of other drugs. Referring to the article ${ }^{47}$ published by domestic researchers last year, it is possible to obtain information on dosage, duration, and termination of hypnotic medications. Forth, this guideline does not include precautions for special populations, such as pregnant women or patients with impairment in the liver or kidneys, although it includes some of the precautions about the elderly. In this regard, clinicians should be careful when applying this guideline to special populations. Fifth, since antipsychotics are sometimes prescribed in actual clinical settings, we searched for papers that could be evidence, but there was no solid evidence. Therefore, it is necessary to supplement it after establishing evidence in the future. Lastly, we were unfortunately unable to contain information on sleep measurements and reliability tests through wearable devices or smartphone apps and digital CBT-I. If the guidelines are to be revised, it may be worth considering the inclusion of relevant content. 


\section{Supplementary Materials}

The online-only Data Supplement is available with this article at https://doi.org/10.30773/pi.2020.0146.

\section{Acknowledgments}

This study was conducted with the support of the clinical practice guidelines development project of the Korean Neuropsychiatric Association in 2018.

\section{Conflicts of Interest}

The authors have no potential conflicts of interest to disclose.

\section{Author Contributions}

Conceptualization: Soyoung Youn, Seockhoon Chung. Data curation: Hayun Choi, Soyoung Youn, Yoo Hyun Um, Tae Won Kim, Gawon Ju, Hyuk Joo Lee, Chungsuk Lee, Sang Don Lee, Kyungyeol Bae, Seong Jae Kim, Ji Hyun Lee, Tae Kim. Formal analysis: Hayun Choi, Soyoung Youn, Seockhoon Chung. Funding acquisition: Seockhoon Chung. Investigation: all authors. Methodology: Seockhoon Chung. Project administration: Soyoung Youn, Seockhoon Chung. Resources: all authors. Software: Seockhoon Chung. Supervision: Seockhoon Chung. Validation: Hayun Choi, Seockhoon Chung. Visualization: Hayun Choi, Seockhoon Chung. Writing_original draft: Hayun Choi. Writing_review \& editing: Hayun Choi, Seockhoon Chung.

\section{ORCID iDs}

$\begin{array}{ll}\text { Hayun Choi } & \text { https://orcid.org/0000-0002-8404-8006 } \\ \text { Soyoung Youn } & \text { https://orcid.org/0000-0003-4983-4017 } \\ \text { Yoo Hyun Um } & \text { https://orcid.org/0000-0002-3403-4140 } \\ \text { Tae Won Kim } & \text { https://orcid.org/0000-0003-2689-202X } \\ \text { Gawon Ju } & \text { https://orcid.org/0000-0002-5358-4578 } \\ \text { Hyuk Joo Lee } & \text { https://orcid.org/0000-0002-3569-9603 } \\ \text { Chungsuk Lee } & \text { https://orcid.org/0000-0003-3984-0165 } \\ \text { Sang Don Lee } & \text { https://orcid.org/0000-0001-8393-9846 } \\ \text { Kyungyeol Bae } & \text { https://orcid.org/0000-0003-0064-4554 } \\ \text { Seong Jae Kim } & \text { https://orcid.org/0000-0003-1699-9560 } \\ \text { Ji Hyun Lee } & \text { https://orcid.org/0000-0001-6096-7561 } \\ \text { Tae Kim } & \text { https://orcid.org/0000-0003-0201-5401 } \\ \text { Seockhoon Chung } & \text { https://orcid.org/0000-0002-9798-3642 }\end{array}$

\section{REFERENCES}

1. Sateia MJ. International classification of sleep disorders. Chest 2014; 146:1387-1394.

2. American Psychiatric Association. Diagnostic and Statistical Manual of Mental Disorders (DSM- $\left.5^{\circledR}\right)$. Washington DC: American Psychiatric Pub; 2013.

3. Sateia MJ, Buysse DJ, Krystal AD, Neubauer DN, Heald JL. Clinical practice guideline for the pharmacologic treatment of chronic insomnia in adults: an American Academy of Sleep Medicine Clinical Practice Guideline. J Clin Sleep Med 2017;13:307-349.

4. Riemann D, Baglioni C, Bassetti C, Bjorvatn B, Dolenc Groselj L, Ellis JG, et al. European guideline for the diagnosis and treatment of insomnia. J Sleep Res 2017;26:675-700.

5. Chung S, Park B, Yi K, Lee J. Pattern of hypnotic drug prescription in South Korea: health insurance review and assessment service-national sample. Sleep Med Res 2013;4:51-55.

6. The Korean Medical Guidelines. Available at: https://www.guideline. or.kr/. Accessed February 28, 2018.

7. Adaptation Process for Developing Korean Clinical Practice Guidelines. Available at: https://www.guideline.or.kr/evaluation/file/koreaguide.pdf. Accessed March 10, 2018.

8. Huang X, Lin J, Demner-Fushman D, editors. Evaluation of PICO as a knowledge representation for clinical questions. AMIA annual symposium proceedings. American Medical Informatics Association, 2006, p. 359.

9. Qaseem A, Kansagara D, Forciea MA, Cooke M, Denberg TD. Clinical Guidelines Committee of the American College of Physicians. Management of chronic insomnia disorder in adults: a clinical practice guideline from the American college of physicians. Ann Intern Med 2016;165:125-133.

10. The AGREE II Instrument. Available at: http://www.agreetrust.org. Accessed March 10, 2018.

11. Atkins D, Best D, Briss PA, Eccles M, Falck-Ytter Y, Flottorp S, et al. Grading quality of evidence and strength of recommendations. BMJ 2004;328:1490.

12. Guyatt GH, Oxman AD, Vist GE, Kunz R, Falck-Ytter Y, Alonso-Coello P, et al. GRADE: an emerging consensus on rating quality of evidence and strength of recommendations. BMJ 2008;336:924-926.

13. Littner M, Hirshkowitz M, Kramer M, Kapen S, Anderson WM, Bailey $\mathrm{D}$, et al. Practice parameters for using polysomnography to evaluate insomnia: an update. Sleep 2003;26:754-760.

14. Ancoli-Israel S, Cole R, Alessi C, Chambers M, Moorcroft W, Pollak $\mathrm{CP}$. The role of actigraphy in the study of sleep and circadian rhythms. Sleep 2003;26:342-392.

15. Morin CM, Belleville G, Bélanger L, Ivers H. The Insomnia Severity Index: psychometric indicators to detect insomnia cases and evaluate treatment response. Sleep 2011;34:601-608.

16. Buysse DJ, Reynolds CF, Monk TH, Berman SR, Kupfer DJ. The Pittsburgh Sleep Quality Index: a new instrument for psychiatric practice and research. Psychaitry Res 1989;28:193-213.

17. Morin CM, Vallieres A, Ivers H. Dysfunctional beliefs and attitudes about sleep (DBAS): validation of a brief version (DBAS-16). Sleep 2007;30:1547-1554.

18. Johns MW. A new method for measuring daytime sleepiness: the Epworth sleepiness scale. Sleep 1991;14:540-545.

19. Glenville M, Broughton R. Reliability of the Stanford Sleepiness Scale compared to short duration performance tests and the Wilkinson $\mathrm{Au}$ ditory Vigilance Task. Adv Biosci 1978;21:235-244.

20. Chung F, Yegneswaran B, Liao P, Chung SA, Vairavanathan S, Islam S, et al. STOP questionnaire: a tool to screen patients for obstructive sleep apnea. Anesthesiology 2008;108:812-821.

21. Ong TH, Raudha S, Fook-Chong S, Lew N, Hsu AA. Simplifying STOP-BANG: use of a simple questionnaire to screen for OSA in an Asian population. Sleep Breath 2010;14:371-376.

22. Netzer NC, Stoohs RA, Netzer CM, Clark K, Strohl KP. Using the Berlin Questionnaire to identify patients at risk for the sleep apnea syndrome. Ann Intern Med 1999;131:485-491.

23. Horne JA, Ostberg O. A self-assessment questionnaire to determine morningness-eveningness in human circadian rhythms. Int J Chronobiol 1976;4:97-110.

24. Spoormaker VI, Verbeek I, van den Bout J, Klip EC. Initial validation of the SLEEP-50 questionnaire. Behav Sleep Med 2005;3:227-246.

25. Morgenthaler T, Kramer M, Alessi C, Friedman L, Boehlecke B, Brown T, et al. Practice parameters for the psychological and behavioral treatment of insomnia: an update. An American academy of sleep medicine report. Sleep 2006;29:1415-1419.

26. Riemann D, Perlis ML. The treatments of chronic insomnia: a review of benzodiazepine receptor agonists and psychological and behavioral therapies. Sleep Med Rev 2009;13:205-214.

27. Morin CM. Cognitive-behavioral approaches to the treatment of insomnia. J Clin Psychiatry 2004;65 (Suppl 16):33-40.

28. Sadock B, Ruiz P. Kaplan \& Sadock's Synopsis of Psychiatry: Behavioral Sciences. New York: Walters Kluwer; 2015.

29. Billioti de Gage S, Pariente A, Begaud B. Is there really a link between benzodiazepine use and the risk of dementia? Expert Opin Drug Saf 2015;14:733-747.

30. Flurazepam, triazolam, flunitrazepam, and brotizolam. Available at: 
www.druginfo.co.kr. Accessed August 10, 2018.

31. French DD, Spehar AM, Campbell RR, Palacios P, Coakley RW, Coblio $\mathrm{N}$, et al. Outpatient Benzodiazepine Prescribing, Adverse Events, and Costs In: Henriksen K, Battles JB, Marks ES, Lewin DI, Editors. Advances in Patient Safety: from Research to Implementation (Volume 1: Research Findings). Rockville, MD: Agency for Healthcare Research and Quality, 2005, p.185-198.

32. MacFarlane J, Morin CM, Montplaisir J. Hypnotics in insomnia: the experience of zolpidem. Clin Ther 2014;36:1676-1701.

33. Buscemi N, Vandermeer B, Friesen C, Bialy L, Tubman M, Ospina M, et al. The efficacy and safety of drug treatments for chronic insomnia in adults: a meta-analysis of RCTs. J Gen Intern Med 2007;22:13351350.

34. Wong CK, Marshall NS, Grunstein RR, Ho SS, Fois RA, Hibbs DE, et al. Spontaneous adverse event reports associated with zolpidem in the United States 2003-2012. J Clin Sleep Med 2017;13:223-234.

35. FDA requires lower dosing of zolpidem. Med Lett Drugs Ther 2013; 55:5.

36. Wade AG, Ford I, Crawford G, McMahon AD, Nir T, Laudon M, et al. Efficacy of prolonged release melatonin in insomnia patients aged 5580 years: quality of sleep and next-day alertness outcomes. Curr Med Res Opin 2007;23:2597-2605.

37. Yeung WF, Chung KF, Yung KP, Ng TH. Doxepin for insomnia: a systematic review of randomized placebo-controlled trials. Sleep Med Rev 2015;19:75-83.

38. Jaffer KY, Chang T, Vanle B, Dang J, Steiner AJ, Loera N, et al. Trazo- done for insomnia: a systematic review. Innov Clin Neurosci 2017;14: 24-34.

39. Stahl SM. Mechanism of action of trazodone: a multifunctional drug. CNS spectr 2009;14:536-546.

40. Generali JA, Cada DJ. Trazodone: insomnia (adults). Hosp Pharm 2015;50:367-369.

41. Yi XY, Ni SF, Ghadami MR, Meng HQ, Chen MY, Kuang L, et al. Trazodone for the treatment of insomnia: a meta-analysis of randomized placebo-controlled trials. Sleep Med 2018;45:25-32.

42. Vande Griend JP, Anderson SL. Histamine-1 receptor antagonism for treatment of insomnia. J Am Pharm Assoc 2012;52:e210-e219.

43. Morin CM, Koetter U, Bastien C, Ware JC, Wooten V. Valerian-hops combination and diphenhydramine for treating insomnia: a randomized placebo-controlled clinical trial. Sleep 2005;28:1465-1471.

44. Chung S, Youn S, Yi K, Park B, Lee S. Sleeping pill administration time and patient subjective satisfaction. J Clin Sleep Med 2016;12:57-62.

45. Youn S, Han CC, Park B, Lee S, Yi K, Chung S. The effects of the new guidance 'take your sleeping pills $7 \mathrm{~h}$ before your wake-up time': a pilot study. Sleep Biol Rhythms 2016;14:397-404.

46. Chung S, Youn S, Park B, Lee S, Kim C. The effectiveness of prolongedrelease melatonin in primary insomnia patients with a regular sleepwake cycle. Sleep Med Res 2016;7:16-20.

47. Nam Y, Cho C, Lee Y, Lee H. Development of safety usage guidelines for sedative hypnotics using the Delphi technique. Sleep Med Psychophysiol 2019;26:2. 


\begin{tabular}{|c|c|c|}
\hline Year & Issuer & Title \\
\hline 2004 & NICE & Guidance on the use of zaleplon, zolpidem and zopiclone for the short-term management of insomnia ${ }^{1}$ \\
\hline 2006 & AASM & $\begin{array}{l}\text { Practice parameters for the psychological and behavioral treatment of insomnia: an update. An American Academy of } \\
\text { Sleep Medicine report }{ }^{2}\end{array}$ \\
\hline 2008 & AASM & Clinical guideline for the evaluation and management of chronic insomnia in adults ${ }^{3}$ \\
\hline 2009 & Japan & The guidelines for the evaluation and management of insomnias in Japanese primary care ${ }^{4}$ \\
\hline 2010 & BSA & New guideline for diagnosis and treatment of insomnia ${ }^{5}$ \\
\hline 2014 & NGC & Clinical guideline for the treatment of primary insomnia in middle aged and older adults ${ }^{6}$ \\
\hline 2015 & NICE & Hypnotics ${ }^{7}$ \\
\hline 2015 & TOP & Assessment to management of adult insomnia ${ }^{8}$ \\
\hline 2016 & $\mathrm{ACP}$ & $\begin{array}{l}\text { Management of chronic insomnia disorder in adults: a clinical practice guideline from the American College of } \\
\text { Physicians }{ }^{9}\end{array}$ \\
\hline 2016 & NHS & Guidelines for treatment of primary insomnia ${ }^{10}$ \\
\hline 2017 & AASM & $\begin{array}{l}\text { Clinical practice guideline for the pharmacologic treatment of chronic insomnia in adults: an American Academy of Sleep } \\
\text { Medicine clinical practice guideline }^{11}\end{array}$ \\
\hline 2017 & ESRS & European guideline for the diagnosis and treatment of insomnia ${ }^{12}$ \\
\hline
\end{tabular}

NICE: national institute for health and care excellence, GIN: Guidelines International network, AASM: American association of sleep medicine, BSA: the Brazilian Sleep Association, TOP: toward optimized practice, NGC: National Guideline Clearinghouse, ACP: American college of physician, NHS: national health system, ESRS: European Sleep Research Society 
Supplementary Table 2. Population, Intervention, Professionals, Outcomes and Health Settings (PIPOH $)^{13}$

\begin{tabular}{lll}
\hline \multicolumn{1}{c}{ PIPOH } & \multicolumn{1}{c}{ Division } & \multicolumn{1}{c}{ Contents } \\
\hline Population & Symptom & Insomnia \\
Intervention & Assessment & Evaluation of sleep structure \\
& Diagnosis & Including methods for differential diagnosis of insomnia \\
& Treatment & Pharmacological treatment \\
& & Non-pharmacological treatment \\
Professions & Clinicians (general practitioners, neuropsychiatrists, neurologists, etc.) \\
Outcome & Patients & Insomnia symptom control and improvement of quality of life \\
& System & Improvement of the appropriateness of assessment, diagnosis and treatment of insomnia disorder \\
Healthcare setting & Medical institutions & Primary medical institutions, outpatient treatment institutions, and inpatient treatment institutions
\end{tabular}


Supplementary Table 3. Evaluation system of the ACP guideline (2016) ${ }^{9}$

\begin{tabular}{lcc}
\hline \multirow{2}{*}{ Quality of evidence } & \multicolumn{2}{c}{ Strength of recommendation } \\
\cline { 2 - 3 } & $\begin{array}{c}\text { Benefits clearly outweigh risks and burden or risks } \\
\text { and burden clearly outweigh benefits }\end{array}$ & Benefits finely balanced with risks and burden \\
\hline High & Strong & Weak \\
Moderate & Strong & Weak \\
Low & Strong & Weak \\
& Insufficient evidence to determine net benefits or risks & \\
\hline
\end{tabular}

*adopted from the classification developed by the GRADE (Grading of Recommendations Assessments, Development, and Evaluation) workgroup. ACP: American College of Physicians 


\begin{tabular}{|c|c|}
\hline Quality of evidence & Definition \\
\hline High quality & Further research is very unlikely to change our confidence in the estimate effect \\
\hline Moderate quality & $\begin{array}{l}\text { Further research is likely to have an important impact on our confidence in the estimate of effect and may change the } \\
\text { estimate }\end{array}$ \\
\hline Low quality & $\begin{array}{l}\text { Further research is very likely to have an important impact on our confidence in the estimate of effect and is likely to } \\
\text { change the estimate }\end{array}$ \\
\hline Very low quality & Any estimate of effect is uncertain \\
\hline
\end{tabular}




\begin{tabular}{cl}
\hline Quality of evidence & Definition \\
\hline High & Corresponds to a high level of certainty that the estimate of the effect lies close to that of the true effect \\
Moderate & $\begin{array}{c}\text { Corresponds to a moderate level of certainty in the effect estimate; the estimate of the effect is likely to be close to the } \\
\text { true effect, but there is a possibility that it is substantially different }\end{array}$ \\
Low & $\begin{array}{c}\text { Corresponds to a low level of certainty in the effect estimate; the estimate of the effect may be substantially different } \\
\text { from the true effect } \\
\text { Very low }\end{array} \quad \begin{array}{l}\text { Corresponds to very little certainty in the effect estimate; the estimate of the effect is likely to be substantially different } \\
\text { from the true effect }\end{array}$
\end{tabular}

*adopted from the classification developed by the GRADE (Grading of Recommendations Assessments, Development, and Evaluation) workgroup. AASM: American Academy of Sleep Medicine 
Supplementary Table 6. Evaluation system in this guideline $(A)$ and conversion tables between guidelines for strength of recommendation (B) and quality of evidence $(C)$

\begin{tabular}{ccc}
\hline (A) Evaluation system & \multicolumn{2}{c}{ Strength of recommendation } \\
\hline \multirow{2}{*}{ Quality of evidence } & $\begin{array}{c}\text { Benefits clearly outweigh risks and burden or risks } \\
\text { and burden clearly outweigh benefits }\end{array}$ & Benefits finely balanced with risks and burden \\
\hline High & Strong & Weak \\
Moderate & Strong & Weak \\
Low & Strong & Weak \\
& Insufficient evidence to determine net benefits or risks & \\
\hline
\end{tabular}

(B) Conversion table between guidelines - strength of recommendation

\begin{tabular}{lcccc}
\hline & ACP guideline $(2016)^{9}$ & ESRS guideline (2017) & AASM guideline (2017) & Korean version guideline (2019) \\
\hline Strength of & Strong & Strong & Strong & Strong \\
recommendation & Weak & Weak & Weak & Weak \\
\hline
\end{tabular}

(C) Conversion table between guidelines - quality of evidence

\begin{tabular}{lllll}
\hline & ACP guideline $(2016)^{9}$ & ESRS guideline $(2017)^{12}$ & AASM guideline $(2017)^{11}$ & Korean version guideline (2019) \\
\hline \multirow{4}{*}{ Quality of evidence } & High & High & High & High \\
& Moderate & Moderate & Moderate & Moderate \\
& Low & Low & Low & Low \\
& & Very low & Very low & \\
\hline
\end{tabular}

ACP: American College of Physicians, ESRS: European Sleep Research Society, AASM: American Academy of Sleep Medicine

\section{REFERENCES}

1. Guidance on the use of zaleplon, zolpidem and zopiclone for the short-term management of insomnia. Available at: https://www.nice.org.uk/guidance/ ta77. Accessed May 3, 2018.

2. Morgenthaler T, Kramer M, Alessi C, Friedman L, Boehlecke B, Brown T, et al. Practice parameters for the psychological and behavioral treatment of insomnia: an update. An american academy of sleep medicine report. Sleep 2006;29:1415-1419.

3. Schutte-Rodin S, Broch L, Buysse D, Dorsey C, Sateia M. Clinical guideline for the evaluation and management of chronic insomnia in adults. J Clin Sleep Med 2008;4:487-504.

4. Yamadera W, Itoh H. The guidelines for the evaluation and management of insomnias in Japanese primary care. Jpn J Clin Med 2009;67:1469-1474.

5. New guideline for diagnosis and treatment of insomnia. Available at: https://www.scielo.br/scielo.php?pid=S0004-282X2010000400038\&script=sci_arttext. Accessed May 3, 2018

6. Clinical guideline for the treatment of primary insomnia in middle aged and older adults. Available at: https://www.multiplechronicconditions.org/assets/ pdf/Insomnia\%20Guidelines/AHRQ_2014_Clinical_guideline_for_the_treatment_of_primary_insomnia_in_middle-aged_and_older_adults.pdf. Accessed May 3, 2018.

7. Hypnotics. Available at: https://www.nice.org.uk/advice/KTT6/chapter/Evidence-context. Accessed May 3, 2018.

8. Assessment to management of adult insomnia. Available at: https://top.albertadoctors.org/ CPGs/Lists/CPGDocumentList/Adult-Insomnia-CPG.pdf. Accessed May 3, 2018.

9. Qaseem A, Kansagara D, Forciea MA, Cooke M, Denberg TD; Clinical Guidelines Committee of the American College of Physicians. Management of chronic insomnia disorder in adults: a clinical practice guideline from the American College of physicians. Ann Intern Med 2016;165:125-133.

10. Guidelines for treatment of primary insomnia. Available at: www.southernhealth.nhs.uk. Accessed May 3, 2018.

11. Sateia MJ, Buysse DJ, Krystal AD, Neubauer DN, Heald JL. Clinical practice guideline for the pharmacologic treatment of chronic insomnia in adults: an American academy of sleep medicine clinical practice guideline. J Clin Sleep Med 2017;13:307-349.

12. Riemann D, Baglioni C, Bassetti C, Bjorvatn B, Dolenc Groselj L, Ellis JG, et al. European guideline for the diagnosis and treatment of insomnia. J Sleep Res 2017;26:675-700.

13. Adaptation Process for Developing Korean Clinical Practice Guidelines. Available at: https://www.guideline.or.kr/evaluation/file/koreaguide.pdf. Accessed March 10, 2018. 